\title{
GESUNDHEITSERZIEHUNG ZUR KRANKHEITSPRÄVENTION: EINE LITERATURÜBERSICHT
}

\section{ARTIKEL ÜBERPRÜFEN}

GITIRANA, José Valdeci Almeida1', FONSECA, Rosa Maria Batista Pinheiro da², PILONETO, Fábio Marmentini ${ }^{3}$, BEVILAQUA, Luis Felipe Gaia ${ }^{4}$, ASSIS, Ingrid de ${ }^{5}$, CARDOSO, Ronald de Oliveira ${ }^{6}$

GITIRANA, José Valdeci Almeida. Et al. Gesundheitserziehung zur Krankheitsprävention: eine Literaturübersicht. Revista Científica Multidisciplinar Núcleo do Conhecimento. Jahr. 06, Hrsg. 11, Vol. 08, S. 134-147. Oktober 2021. ISSN: 2448-0959, Zugangslink:

https://www.nucleodoconhecimento.com.br/gesundheit/gesundheitserziehung, DOI: 10.32749/nucleodoconhecimento.com.br/gesundheit/gesundheitserziehung

\section{ZUSAMMENFASSUNG}

Derzeit werden öffentliche Politiken, Regierungsmaßnahmen und verschiedene Programme zur Gesundheitserziehung (ES) zugunsten der Gesundheit der Bevölkerung durchgeführt. In Bezug auf das Thema ES ist dies ein facettenreiches Thema, das mit verschiedenen persönlichen Vorstellungen konvergiert, sei es im Bereich Bildung oder Gesundheit, was zu unterschiedlichen Verständnissen führen kann. Aufgrund der Komplexität des Themas tritt das Problem aufgrund des mangelnden Verständnisses dessen auf, was wirklich bevölkerungsbezogene ES ist, wodurch die Bedeutung von ES für Präventionsmaßnahmen gegen verschiedene Krankheiten aufgelöst wird, was zu hohen Haushaltskosten im öffentlichen

\footnotetext{
${ }^{1}$ Abschluss in Pharmazie, Postgraduiertenstudium in klinischer Analyse, derzeit medizinisch.

${ }^{2}$ Medizin.

${ }^{3}$ Studium der Betriebswirtschaftslehre. Absolvent des Außenhandels, derzeit Medizinstudent.

${ }^{4}$ Abschluss in Lebensmitteltechnik, Postgraduiertenstudium in industrieller Verfahrenstechnik, CBA in Betriebswirtschaft und derzeit Medizinstudent.

${ }^{5}$ Medizin.

${ }^{6}$ Medizin.

RC: 102790

Verfügbar in: 
Gesundheitswesen führt. In Anbetracht dieses Zusammenhangs stellt sich in diesem Artikel die Hauptfrage: Wie kann Gesundheitserziehung zur Prävention von Krankheiten in der Bevölkerung beitragen? Die Studie zielte darauf ab, das ES zur Krankheitsprävention (PD) vorzustellen, sich in seinen Konzepten zu verzweigen und die rechtlichen Grundlagen aufzuzeigen, die das ES für die Bevölkerung gewährleisten. Zu diesem Zweck wurde die Studie durch eine Literaturrecherche der Suchseite "Google Scholar", "Scielo" und "PubMed" durchgeführt. Durch die Deskriptoren: Gesundheitserziehung für die Bevölkerung, wobei Studien ausgewählt wurden, die das ES mit Schwerpunkt auf PD präsentierten. Es wird geschlussfolgert, dass ES Bildung zur Selbsterkenntnis und Reflexion über die eigene Gesundheit ist, sowie das volle Bewusstsein, dass etwas nicht stimmt, und schreibt der Person die kritische Reflexion ihrer Gewohnheiten und Präventionsmaßnahmen zu. Es wird durch den schulischen Lehrplan ab der Grundbildung sowie durch staatliche Programme in Gesundheitszentren angewendet, um die Gesellschaft für Präventionsmaßnahmen und die Förderung einer besseren Lebensqualität zu sensibilisieren und so die Ausbreitung von Krankheiten zu verhindern.

Schlüsselwörter: Gesundheitserziehung, Krankheitsprävention, Gesundheitsförderung, Bevölkerung.

\section{EINLEITUNG}

Gesundheitserziehung (ES) ist eine soziale Praxis, deren Lernprozess zur Bildung eines kritischen persönlichen Bewusstseins für Gesundheitsprobleme selbst beiträgt und den Bürger dazu bringt, über ihre Realität nachzudenken und daraus Lösungen und Organisation zugunsten ihrer Gesundheit und kollektiven Gesundheit zu suchen. zu einer Gesundheitsförderung (PS) (BISCARDE; PEREIRA-SANTOS( SILVA, 2014).

Die Ursprünge und Konzeptionen von PS begannen mit dem Aufkommen von ES im frühen 20 ). Damals wurde die Bedeutung von PS den Maßnahmen der Hochschulbildung zugeschrieben, die auf eine bessere Lebensqualität abzielten. ES 
hat jedoch derzeit eine verstärkte Eigenschaft, da sie als eines der wichtigsten Instrumente für die Durchführbarkeit von PS gilt und die Entwicklung der Eigenverantwortung und die Prävention von Krankheiten (PD) leitet (LOPES; SARAIVA; XIMENES, 2010; FALKENBERG et al ., 2014; SPERANDIO; SPERANDIO, 2018).

Obwohl die ES als eine der Maßnahmen für die PD (JANINI; BESSLER; VARGAS, 2015; SPERANDIO; SPERANDIO, 2018), ist es ein facettenreiches Thema, das mit verschiedenen persönlichen Vorstellungen konvergiert, sei es für Menschen im Bildungsbereich oder im Bereich der Gesundheit, was zu unterschiedlichen Verständnissen führen kann. (SCHALL; STRUCHINER, 1999).

Aufgrund der Komplexität des Themas entsteht das Problem aufgrund des fehlenden Verständnisses dessen, was ES wirklich ist, das sich an die Bevölkerung richtet und die Bedeutung von ES für präventive Maßnahmen gegen verschiedene Krankheiten auflöst. Aus diesem Grund werden viele Krankheiten, die vermieden werden könnten, oft nachgewiesen, die große Auswirkungen auf den öffentlichen Haushalt haben. Daher konnte diese Studie hervorragend die Frage beantworten: Wie kann Gesundheitserziehung zur Prävention von Krankheiten in der Bevölkerung beitragen? Das allgemeine Ziel war es, durch eine Literaturrecherche darzustellen, wie ES PD fördern kann, indem es ihre Konzepte aufgreift und die rechtlichen Grundlagen hervorhebt, die der Bevölkerung eine höhere Bildung sichern.

Zu diesem Zweck wurde die Studie durch eine Literaturrecherche der Suchseite "Google Scholar", "Scielo" und "PubMed" durchgeführt. Durch die Deskriptoren: Gesundheitserziehung; Gesundheitserziehung als Krankheitsprävention; Gesundheitserziehung für die Bevölkerung. Als Weg der Aufnahme wurden zwischen 2018 und 2020 Artikel und Dokumente ausgewählt, die die notwendige Struktur bilden, um das gewünschte Ziel zu erreichen. Anschließend wurden 20 Artikel gefunden, von denen 8 ausgewählt wurden. Die Artikel, die das Ziel und die Leitfrage beantworteten und innerhalb des vorgeschlagenen Zeitrahmens lagen, wurden 
einbezogen. Studien, die sich nur an Angehörige der Gesundheitsberufe oder im Gesundheitsbereich qualifizierte Studenten richteten, wurden ausgeschlossen.

Das Thema ist heute relevant, da ES die Reflexionsfähigkeit der Bevölkerung zur Bekämpfung verschiedener Krankheiten beeinflussen kann, was zu besseren Lebensgewohnheiten führt. Es kann für Laien oder für Studenten und Fachleute im akademischen Bereich relevant sein und als Quelle für zukünftige Studien dienen, da es das Konzept von ES für die Bevölkerung als untrennbar mit der PD aufklärt und eine Sprache bietet, die leicht zu verstehen ist.

\section{DIE UNTRENNBARKEIT VON GESUNDHEITSERZIEHUNG UND KRANKHEITSPRÄVENTION}

ES wird auf unterschiedliche Weise geplant und angewendet, eine solche Lehrmodalität kann sich an Studierende im Gesundheitsbereich richten; für Angehörige der Gesundheitsberufe; für die Schulbildung und für die Bevölkerung im Allgemeinen. In der Tat wird es auf unterschiedliche Weise verstanden und entspricht der kulturellen und sozialen Bildung jedes Einzelnen. Die vorliegende Studie betont jedoch, dass ES für die Bevölkerung untrennbar mit PD verbunden ist, da ES "[...] mit den allgemeinen Zielen der öffentlichen Gesundheit verbunden ist: Krankheitsprävention, Schutz und Förderung der Gesundheit des Einzelnen, der Familie und der Gemeinschaft" (PIMONT, 1997, S. 17).

Um das Verständnis dieser Untrennbarkeit besser zu kontextualisieren, ist es wichtig hervorzuheben, dass die ES eine ist:

Processo educativo de construção de conhecimentos em saúde que visa à apropriação temática pela população [...]. Conjunto de práticas do setor que contribui para aumentar a autonomia das pessoas no seu cuidado e no debate com os profissionais [...] a fim de alcançar uma atenção de saúde de acordo com suas necessidades. (BRASIL, 2012, p. 19).

Wie im "Thematischen Glossar: Arbeitsmanagement und Gesundheitserziehung" vorgestellt, bewirkt das ES die wünschenswerte Autonomie, damit sich die Menschen 
effektiv ausdrücken können, um ihre spezifischen Bedürfnisse in ihrer klinischen Versorgung nachzuweisen und zur Versorgung des Angehörigen der Gesundheitsberufe beizutragen, um die Krankheiten umgehend zu behandeln und andere sekundäre Krankheiten zu verhindern. Ergänzend zu dieser Definition wird in dem Dokument der Nationale Gesundheitsstiftung (FUNASA) Brasil (2007) dargestellt, dass ES eine soziale Praxis ist, die bekräftigt, dass ihre Absicht in eine Transformation für jede Art von Person "[...] bricht mit dem Paradigma der statischen Konzeption von Bildung als Transfer von Wissen, Fähigkeiten und Fertigkeiten". (BRASIL, 2007, p. 20).

Pimont (1997, S. 15) fasst verschiedene Konzepte zusammen und analysiert sie und betont, dass ES darauf abzielt, Folgendes zu bieten:

1) A consciência do direito à saúde, dentro do grau de desenvolvimento do grupo social no qual vive o indivíduo; 2) $\mathrm{O}$ conhecimento sobre saúde; 3) O conhecimento dos serviços para a saúde que a comunidade possua e sua consequente utilização; 4) O conhecimento e a utilização das práticas de saúde.

Der Autor stellt jedoch fest, dass es für die Umwandlung dieser Lehre in Wissen zur Förderung von PS und PD notwendig ist, dass eine Planung strukturiert ist, die eine angemessene pädagogische Methodik aus Hilfsinstrumenten abschwächt, die dann zu einem konstanten Bildungsprozess führen kann.

Nach Altuve (1996) Damit ES zu Wissen wird, wird die Gemeinschaftsorganisation bereits zu einer Methode, die sozialen Wandel hervorruft, da seine Anwendung inmitten von Bemühungen und Techniken zur Einleitung und Förderung eines bedeutenden sozialen Wandels führt, der alle sozialen Sektoren umfasst, wie z. politischer und bildungspolitischer Ebene.

Daher kann ES nach Ansicht der zitierten Autoren untrennbar mit PD auf die Bevölkerung verbunden sein, wenn es methodisch übertragen wird. Die vorliegende Studie beabsichtigt jedoch nicht, diese Methoden zu vertiefen, sondern auch ES als PD für die Bevölkerung zu konzeptualisieren. 


\subsection{RECHTSGRUNDLAGEN FÜR DIE GESUNDHEITSERZIEHUNG DER BEVÖLKERUNG}

Mit einem Schwerpunkt auf ES in Brasilien wurde im August 1971 das Gesetz 5692 geschaffen, das den Lehrplan der Primar- und Sekundarstufe neu formuliert und eine gesundheitsorientierte Erziehung gemäß Artikel 7 umsetzt:

Art. $7^{0}$ Será obrigatória a inclusão de Educação Moral e Cívica, Educação Física, Educação Artística e Programas de Saúde nos currículos plenos dos estabelecimentos de ํㅡ e $2^{\circ}$ graus, observado quanto à primeira o disposto no Decreto-Lei n. 369, de 12 de setembro de 1969. (Vide Decreto no 69.450, de 1971)

Spätere Programme wie "Saúde na escola" wurden als intersektorale Strategie im Schulraum und seiner Umgebung entwickelt, die auf PS und Lebensqualität ausgerichtete Maßnahmen zusammen mit Reflexionen über die PD und das Wissen über das Gesundheit Unic-System (SUS) artikuliert (BRASIL, 2012). Dieser Kontext lässt sich dadurch verdeutlichen, dass Maßnahmen für ES lebenslang, ab der ersten Schulstufe, umgesetzt werden, damit die Menschen nach und nach ein kritisches, reflektiertes und bewusstes Empfinden entwickeln.

Durch die positive Durchstufung des Umfangs von Programmen, die auf ES und PD abzielen, wurden unterschiedliche Gesetze geschaffen, gemäß den in Tabelle 1 unten dargestellten Rechtsgrundlagen: 
Tabelle 1 - Rechtsgrundlagen der Gesundheitserziehung.

\begin{abstract}
A Lei n. ${ }^{\circ}$ 8.080/1990, que regulamenta o SUS, em seu Capítulo I, Art. $5^{\circ}$, Item III, define como um dos objetivos e atribuições deste Sistema: "a assistência às pessoas por intermédio de ações de promoção, proteção e recuperação da saúde, com a realização integrada das ações assistenciais e das atividades preventivas". A mesma Lei, em seu Capítulo II, Artigo 7_ ${ }^{\circ}$, Item VIII, estabelece a
\end{abstract} "participação da comunidade."

A Lei $\mathbf{N}^{\circ} \mathbf{8 . 1 4 2}$, de 28 de dezembro de 1990, define a participação da comunidade na gestão SUS e no seu artigo $1^{\circ}$ "as instâncias colegiadas de controle social: I) a Conferência de Saúde, e II) o Conselho de Saúde."

A Norma Operacional Básica - SUS 1/1996 (NOB/96), que redefine as responsabilidades dos estados, do Distrito Federal e da União e consolida as responsabilidades dos municípios, cita como um dos papéis do gestor federal e do estadual a "Educação em Saúde

A Educação em Saúde na Funasa está ancorada em seu Regimento Interno, Portaria GM n ${ }^{\circ} 1.776$ de 8/9/2003, assinada pelo Ministro da Saúde que estabelece as competências da Assessoria de Comunicação e Educação em Saúde-Ascom e seus setores, dentro das competências dos demais setores do órgão. (...)

Lei n. 9.394, de 20 de dezembro de 1996 (1996). Estabelece as diretrizes e bases da educação nacional.

DCN - EF 1988 - Estabelecer as Diretrizes Curriculares Nacionais para o Ensino Fundamental: Saúde articulada à educação e à vida cidadã.

PCN - CN 1988 - Estabelecer as Referências Nacionais Comuns para o Ensino das Ciências Naturais: Saúde como estado de equilíbrio dinâmico do corpo.

PCN - TT Saúde 1988 - Apresentar a saúde como um Tema Transversal: Educação para a saúde

PCN - EM 1999 - Apresentar os Parâmetros Curriculares Nacionais para o Ensino Médio: Degradação ambiental e agravos à saúde humana.

PCN + 2002 - Apresentar Orientações Curriculares complementares aos PCN: Saúde e as condições de vida das pessoas.

OCEM 2006 - Apresentar sugestões para as práticas pedagógicas e organização dos

currículos do Ensino Médio: Ambiente e saúde.

DCNEB 2013 - Estabelecer Orientações Nacionais para a Educação Básica. Saúde como campo de conhecimento e de serviços.

BNCC - $\mathbf{1}^{\mathbf{a}}$ versão 2015 - Estabelecer uma Base Nacional Comum para os currículos da Educação Básica, com versão inicial disponibilizada para consulta pública, estabelecendo cuidado com a saúde da população.

BNCC - 2 ${ }^{\mathrm{a}}$ versão 2016 - Estabelecer uma Base Nacional Comum para os currículos da Educação Básica, com versão elaborada após contribuições da sociedade, para o ambiente e saúde.

BNCC $3^{a}$ versão 2016 - Estabelecer uma Base Nacional Comum para os currículos da Educação Básica com versão disponibilizada após contribuições institucionais, para promoção dos cuidados com a saúde.

BNCC versão final (EI e EF) 2016 - Estabelecer uma Base Nacional Comum para os currículos da Educação Infantil e do Ensino Fundamental, compondo a versão final, com o objetivo de promover os cuidados com a saúde.

BNCC EM 2018 - Estabelecer uma Base Nacional Comum para os currículos do Ensino Médio, promoção do cuidado com a saúde.

Quelle: Angepasst aus Brasil (2012); Sousa, Guimarães und Liebende (2019). 
Der Rahmen stellt die Gesetze vor, in denen einige Bildungsgrundlagen für die Anwendbarkeit von ES in der Schule hervorgehoben werden, auch wenn sich solche Richtlinien auf das schulische Umfeld konzentrieren, leiten die Lehrplanzwecke die Ausbildung von Menschen, die die Bedeutung von ES verstehen und die durch dieses Wissen die Sorge um die Persönliche und Die Gesundheit der Bevölkerung fördern können. auch den Wohltäter der PD zu komponieren.

Heute stellen die Vereinten Nationen das dritte Ziel der "Agenda 2030" vor, und mit globaler Unterstützung soll es durch die Aktion "ein gesundes Leben gewährleisten und das Wohlergehen für alle und alle, in jedem Alter" (SDGs, 2021) unabhängig von sozialer Schicht, Rasse oder Hautfarbe fördern.

Der Platz auf der Agenda 2030 steht im Einklang mit der Perspektive von SHALL (1999), wo sie abgeschwächt, dass die Definition für ES breiter sein muss als nur eine Aktion, die sich aus dem PS ergibt, da sie, die sich auf die Bevölkerung konzentriert, einen umfassenden Prozess der aktiven Beteiligung der gesamten Bevölkerung an ihrem täglichen Leben durchläuft und nicht nur von Menschen, die gefahr gefährdet sind, zu erkranken. dann ein Weg zu sein, Krankheiten für gesunde Menschen zu verhindern, eine bessere Lebensqualität und ein größeres Wohlbefinden für alle zu schaffen.

\subsection{STUDIEN ZUR KRANKHEITSPRÄVENTION DURCH GESUNDHEITSERZIEHUNG}

Als nächstes werden die wichtigsten Erkenntnisse über PD als Ergebnis der Anwendung von ES, die in der wissenschaftlichen Literatur verfügbar sind, zwischen den Jahren 2018 und 2020 vorgestellt. Als nächstes zeigt Tabelle 2 eine Synthese der 8 zuvor ausgewählten Artikel, die in chronologischer Reihenfolge präsentiert werden, wobei die in jeder Studie verwendeten Methoden später erläutert werden. 
Tabelle 2 - Studien im Zusammenhang mit Gesundheitserziehung als Krankheitsprävention.

\begin{tabular}{|c|c|c|}
\hline utor & Ziel & chlussfolgerung \\
\hline $\begin{array}{l}\text { as et } \\
18 \text { ) }\end{array}$ & 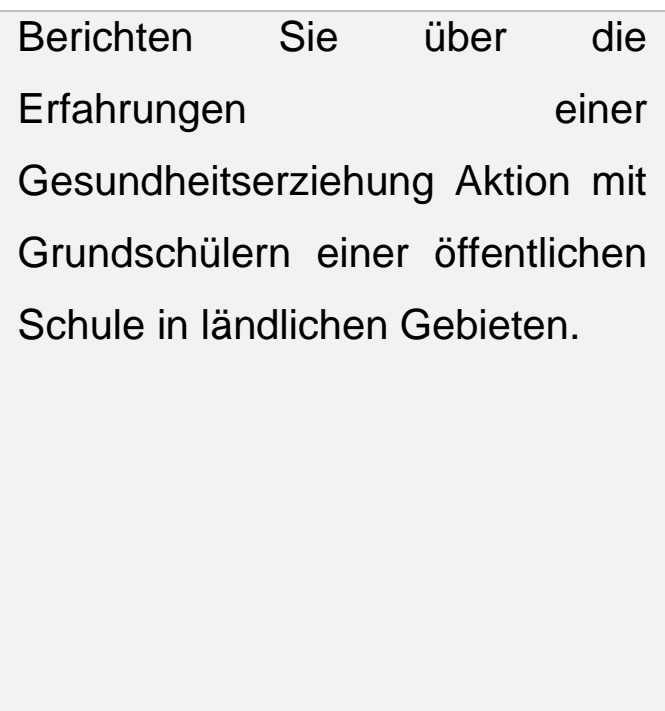 & $\begin{array}{l}\text { Kinder sind angesichts der } \\
\text { sanitären Bedingungen, in denen } \\
\text { sie leben, dem Risiko einer } \\
\text { Kontamination durch Parasiten } \\
\text { ausgesetzt, was die } \\
\text { Notwendigkeit belegt, die } \\
\text { Maßnahmen } \\
\text { Gesundheitserziehung über die } \\
\text { Bedeutung der Verhinderung von } \\
\text { Parasiten auszuweiten. }\end{array}$ \\
\hline $\begin{array}{l}\text { Salci, } \\
\text { Meirelles e } \\
\text { Silva } \\
(2018)\end{array}$ & $\begin{array}{l}\text { Verstehen Sie, wie sich die } \\
\text { Mitglieder der Familie } \\
\text { Gesundheitsstrategie für die } \\
\text { Gesundheitserziehung, das } \\
\text { Management und die Prävention } \\
\text { chronischer Komplikationen von } \\
\text { Diabetes mellitus in der primären } \\
\text { Gesundheitsversorgung } \\
\text { organisieren }\end{array}$ & $\begin{array}{l}\text { Es wird davon ausgegangen, } \\
\text { dass die Praxis der } \\
\text { Gesundheitserziehung für } \\
\text { Menschen mit Diabetes mellitus } \\
\text { in der primären } \\
\text { Gesundheitsversorgung bildet } \\
\text { einen facettenreichen Kontext mit } \\
\text { mehreren Hindernissen für seine } \\
\text { Ausführung. }\end{array}$ \\
\hline $\begin{array}{l}\text { Gomes et } \\
\text { al. (2019) }\end{array}$ & $\begin{array}{l}\text { Bericht über } \\
\text { Aufklärungsmaßnahmen in einer } \\
\text { Gruppe von Männern, um ein } \\
\text { besseres Verständnis von } \\
\text { Peniskrebs sowie Präventions- } \\
\text { und Früherkennungsmaßnahmen } \\
\text { zu erlangen. }\end{array}$ & $\begin{array}{l}\text { Das Wissen über Peniskrebs } \\
\text { muss bei Männern noch besser } \\
\text { herausgearbeitet werden. }\end{array}$ \\
\hline
\end{tabular}

Figueiredo; Führen Sie zur Schaffung einer Public Policies sind essentiell für 


\begin{tabular}{|c|c|c|}
\hline $\begin{array}{l}\text { Antunes e } \\
\text { De Miranda } \\
(2019)\end{array}$ & $\begin{array}{l}\text { öffentlichen Gesundheits } \\
\text { Erziehungspolitik mit spezifischer } \\
\text { Schulbetreuung und } \\
\text { angemessener Überweisung von } \\
\text { Schülern mit Fettleibigkeit an } \\
\text { Gesundheits Einheiten }\end{array}$ & $\begin{array}{l}\text { die Kommunikation und Stärkung } \\
\text { aller Maßnahmen und Leitlinien } \\
\text { in den Bereichen Bildung und } \\
\text { Gesundheit. Interdisziplinarität } \\
\text { wird die gewünschten } \\
\text { Transformationen durch } \\
\text { Präventionsmaßnahmen fördern, } \\
\text { mit dem Ziel, die Beteiligung von } \\
\text { Fettleibigkeit und ihre Folgen in } \\
\text { den jüngeren Altersgruppen } \\
\text { sowie die Verringerung von Herz- } \\
\text { Kreislauf-Erkrankungen in der } \\
\text { frühen Lebensphase und im } \\
\text { Erwachsenenalter zu reduzieren. }\end{array}$ \\
\hline
\end{tabular}

Ricci et al. Berichten Sie über die Die Durchführung von (2019) Erfahrungen von Akademikern Gesundheitserziehung über der Bundesuniversität von Mato sexuell übertragbare Infektionen Grosso do Sul (UFMS) während (STI) während der einer Aktion, die mit der Gruppe Schwangerschaft erwies sich als schwangerer Frauen einer Basic wirksam bei der Bereitstellung Health Unit in Campo Grande - von Dialog, Interaktion, Dem MS (UBS) entwickelt wurde. Austausch von Erfahrungen, Wissen und Erfahrungen und der gemeinsamen Konstruktion von Wissen, das sich dann als wichtig für die Gesundheitsförderung und Krankheitsprävention sowie für die Entwicklung eines kritischen und reflektierenden Bewusstseins für schwangere Frauen über 


\begin{tabular}{|c|c|c|}
\hline & & $\begin{array}{l}\text { ihren Gesundheits- } \\
\text { Krankheitsprozess erwiesen hat. } \\
\text { indem wir sie aktiv im } \\
\text { Lernprozess machen. }\end{array}$ \\
\hline $\begin{array}{l}\text { De Sousa } \\
\text { Gonçalves } \\
\text { et al. } \\
(2020)\end{array}$ & $\begin{array}{l}\text { Um die Arbeit der } \\
\text { Gesundheitserziehung einer } \\
\text { grundlegenden Gesundheits } \\
\text { Einheitin der Gemeinde } \\
\text { Imperatriz }- \text { MA zu kennen. }\end{array}$ & $\begin{array}{l}\text { Es wird angenommen, dass es } \\
\text { wichtig ist, das Wissen und die } \\
\text { Praxis von Fachleuten ständig zu } \\
\text { verbessern, um Maßnahmen zur } \\
\text { Gesundheitserziehung zu } \\
\text { entwickeln. Es ist notwendig, die } \\
\text { Bildungsmaßnahmen innerhalb } \\
\text { des Verantwortungsbereichs } \\
\text { jedes Teams auszuweiten, sowie } \\
\text { eine aktivere Beteiligung aller } \\
\text { Mitglieder des Familienstrategie } \\
\text { Teams sowie der Fachleute des } \\
\text { Familiengesundheitszentrum } \\
\text { (NASF). }\end{array}$ \\
\hline $\begin{array}{l}\text { Lima et al. } \\
(2020)\end{array}$ & $\begin{array}{l}\text { Beschreiben Sie die Erfahrung } \\
\text { als Krankenpflegeschüler in } \\
\text { Gesundheitserziehung Aktivitäten } \\
\text { zur Vorbeugung von Herz- } \\
\text { Kreislauf-Erkrankungen bei } \\
\text { älteren Menschen. }\end{array}$ & $\begin{array}{l}\text { Die Praktiken der } \\
\text { Gesundheitserziehung sind } \\
\text { transformativ und leisten wichtige } \\
\text { Beiträge zur gerontologischen } \\
\text { Krankenpflege, da sie die } \\
\text { Ausübung eines kritisch- } \\
\text { reflektierenden Denkens } \\
\text { ermöglicht und zur Ausbildung } \\
\text { von Fachleuten beiträgt, die sich } \\
\text { der Arbeit in einem Pflegemodell } \\
\text { verschrieben haben, das auf } \\
\text { wissenschaftlichen Erkenntnissen }\end{array}$ \\
\hline
\end{tabular}




\begin{tabular}{|c|c|c|}
\hline & & $\begin{array}{l}\text { basiert und in der Lage ist, die } \\
\text { Bedürfnisse der älteren } \\
\text { Bevölkerung zu erfüllen, die im } \\
\text { Laufe der Jahre gewachsen ist. }\end{array}$ \\
\hline $\begin{array}{l}\text { Costa et al. } \\
(2020)\end{array}$ & $\begin{array}{l}\text { Um eine der } \\
\text { Gesundheitserziehung } \\
\text { melden, geschah im Rahmen der } \\
\text { primären } \\
\text { Gesundheitsversorgung, deren } \\
\text { Vorschlag darin bestand, } \\
\text { Brustkrebs vor der vorherigen } \\
\text { Anerkennung seiner } \\
\text { Manifestationen sowie der } \\
\text { Aufrechterhaltung gesunder } \\
\text { Gewohnheiten zu verhindern, um } \\
\text { die Gesundheit dieser Frauen zu } \\
\text { fördern. }\end{array}$ & $\begin{array}{l}\text { Die entwickelte Maßnahme } \\
\text { diente als Konsolidierungs } \\
\text { Mechanismus der Aspekte, die } \\
\text { die Gesundheitserziehung } \\
\text { ausmachen, und bekräftigte die } \\
\text { grundlegende Rolle der } \\
\text { Krankenpflege im Pflegeprozess. }\end{array}$ \\
\hline
\end{tabular}

Quelle: Der Autor.

Dias et al. (2018), durch einen Erfahrungsbericht mit einer außerklassigen Aktivitätsmethode der curricularen Komponente "Wissenschaftliches Integratorprojekt des Krankenpflege-Grundstudiums" von Fakultät Verde Norte, gehalten im Oktober 2016 in der städtischen Schule Odilon Antunes, im Dorf Poções der Stadt Monte Azul, eine Aktion der Gesundheitserziehung unter Beteiligung von 30 Studenten, 03 Lehrer und 09 Eltern. Die Aktion erfolgte in den Phasen der dialogischen Exposition des Inhalts und in der Anzeige des Videodiskussionsauslösers, der im Gesprächsrad mit dem Publikum diskutiert wurde. So lieferte die Studie vorbeugende Maßnahmen für die Kontamination durch parasitäre Erkrankungen.

Salci; Meirelles und Silva (2018) führte eine qualitative Studie durch, in der komplexes Denken als theoretische Referenz und evaluative Forschung als 
methodische Referenz komponiert wurde. Für die Datenerhebung befragten 38 Angehörige der Gesundheitsberufe Patienten mit Diabetes mellitus. Die Gesundheitsagentur beobachteten und analysierten die Krankenakten der Patienten durch die Triangulation der Daten mit Unterstützung der ATLAS.ti-Software. Die Autoren stellten fest, dass ES je nach Komplexität des facettenreichen Themas primäre und sekundäre PD fördern kann.

Um diese Komplexität zu bestätigen, bestätigt Figueiredo; Antunes und De Miranda (2019) führten eine bibliographische Überprüfung Recherche durch, bei der die explorative Forschungsmethode angewendet wurde. Die Autoren bezogen die Prävention von Komorbiditäten und Herz-Kreislauf-Erkrankungen auf die öffentliche Politik von SSc im schulischen Umfeld. Daher betonten sie, dass die Interdisziplinarität, die SSc einbezieht, die gewünschten Transformationen bei Schülern durch Präventionsmaßnahmen fördern kann, die Herz-KreislaufErkrankungen im Kindes- und Erwachsenenalter verhindern.

Lima et al. (2020) in einer ähnlichen Studie, die sich jedoch an die ältere Öffentlichkeit richtete, beobachteten sie durch einen Erfahrungsbericht, durch die deskriptive Methode, mit einem qualitativen Ansatz, der im "Gesundheitsprogramm für ältere Menschen - PROASI" erlebt wurde, dass die Aktivitäten von ES als Werkzeug bei der Prävention von Herz-Kreislauf-Erkrankungen dienen und ein kritisch-reflexives Denken für ihre eigene Gesundheit und Lebensqualität verursachen.

Gomes et al. (2019) durch die explorative deskriptive Studie der Art des Erfahrungsberichts wandten sie eine Aktivität an, die im März 2018 in einer Kirche am Stadtrand von Belém/Pará durchgeführt wurde. Das teilnehmende Publikum bestand aus dreiundzwanzig Männern im Alter von 18 bis 50 Jahren, die zu Vorträgen mit der Präsentation von Bannern eingereicht wurden; Erklärung von Peniskrebs; Pflege von PD und Demonstration von Penisprothesen. Die Autoren beobachteten durch das Gespräch Rad mit den Teilnehmern, die nur wenige über das Thema Peniskrebs Bescheid wussten. Daher warnten die Autoren vor der 
Bedeutung der Implementierung von ES-Programmen, die auf PD wie Peniskrebs abzielen, da mit Programmen, die auf ES abzielen, diese und andere Krankheiten in der Bevölkerung erheblich reduziert werden können.

Noch im Rahmen der Krebsprävention haben Costa et al. (2020) führte eine deskriptive Studie mit qualitativem Ansatz über die Art des Erfahrungsberichts durch. Die Studie wurde im Krankenhausbereich durchgeführt, der sich in der Peripherie Region der Stadt Belém-PA befindet, in einer Gesundheits Einheit mit Fachleuten, die eine Modalität der Gesundheitsbehandlung fördern, die sich auf die primäre Ebene von Krankheiten konzentriert. Die an die weibliche Öffentlichkeit gerichteten Behandlungen werden in der Umsetzung des "Women's Health Program" konsolidiert, bei dem Patienten mit Schwangerschaftsvorsorge behandelt werden; Geburt; postpartal; Pflege der Familien; Programme zur Vorbeugung von Brust- und Gebärmutterkrebs; IST. Dieses Programm besteht aus Fachleuten aus verschiedenen Gesundheitsbereichen, zusammen mit den verschiedenen Arten von klinischen Analysen für die Annahme von Präventionsmaßnahmen, die die vorherige Diagnose von Pathologien wie Krebs fördern. Mit der Implementierung einer Aufklärungsmaßnahme im Programm folgten Angehörige der Gesundheitsberufe den Patienten in ihren Konsultationen und ähnlich wie in der Studie von Gomes et al. (2019) exponierte Ordner; anatomische Präsentationen der Brust; neben anderen pädagogischen und interaktiven Aktivitäten. Daher erklärten die Autoren, dass die 16 Teilnehmer eine größere Reflexion über die Bedeutung der Selbstversorgung hatten und die Bedeutung von SSc für die Prävention von Brustkrebs verstanden.

In Bezug auf diese zitierte Reflexion von Gomes et al. (2019), Ricci et al. (2019) bestätigen, dass ein solcher Prozess des reflexiven Bewusstseins durch Dialog stattfindet; Interaktion; Erfahrungsaustausch; Wissen und Erfahrungen und die gemeinsame Konstruktion von Wissen, da sich solche Elemente als wichtig für die PS und die PD erwiesen. Diese Beobachtung wurde durch die Studie belegt, die am 15. März 2017 in der ersten Nacht im Besprechungsraum einer Basic Health Unit von Campo Grande - MS - stattfand. Es wurde eine Methodik in strategischer Weise verwendet, die sich aus Praktiken der Lächerlichkeit zusammensetzt, mit dem Ziel 
der Interaktivität der Teilnehmer, so dass sie durch pädagogische Praxis das Verständnis des betreffenden Themas erwerben konnten. Zunächst wurden schwangere Teilnehmerinnen durch Einberufungen von Gemeindevertretern in der Region der Gesundheits Einheit eingeladen. Später Begann die Aktion mit der Eisbrecher Technik "vorbei an der baby", bei der die Teilnehmer mit ihrem Namen, ihrem Gestationsalter und der Wahl des Kindernamens identifiziert wurden. Durch die Dynamik wurden die schwangeren Frauen auf die Krankheiten aufmerksam, die während der Schwangerschaft verhindert werden konnten, sowie auf die PD im Baby selbst.

Damit dieses reflexive Bewusstsein die Familie einbezieht, haben De Sousa Gonçalves et al. (2020), führte eine quantitative Arbeit mit analytischem Ansatz durch, die auf einer retrospektiven Sammlung der von Februar bis September 2019 durchgeführten Maßnahmen von ES basiert. Die Forschung wurde in einer Basisgesundheit Einheit (UBS) der Gemeinde Imperatriz im Bundesstaat Maranhão durchgeführt, in der sie sich aus 3 Familien Gesundheitsstrategie Teams zusammensetzte. Die Daten wurden aus der Dokumentensammlung mit Aufzeichnungen über die Aktionen der ES in der UBS und in den Schulen der Region gesammelt, die von jedem Team im oben genannten Zeitraum abgedeckt und von der Leitung der Gesundheits Einheit zur Verfügung gestellt wurden. Die Autoren analysierten, dass durch ES nicht nur chronische, sexuelle oder infektiöse Erkrankungen verhindert werden können, sondern auch prophylaktische Maßnahmen aus Programmen resultieren können, die diesen Familien helfen.

\section{ENDGÜLTIGE ÜBERLEGUNGEN}

ES kann PD durch öffentliche Politikprogramme fördern, die darauf abzielen, ESBildungsprogramme in ES, UBS, Krankenhaus Netzwerken sowie Gesundheitsämtern umzusetzen. Dafür können seine Konzepte breit präsentiert werden, da ES ein komplexes und facettenreiches Thema ist. 
Aus den Erkenntnissen geht hervor, dass die ES die PD gemeinsam mit der PS für die Bevölkerung anregen kann, wenn diese Zielgruppe durch methodisch geplante und angewandte Aktivitäten unter Einbringung spezifischer Instrumente zur Lernentwicklung eingebunden wird. In Bezug auf PD wurden in den Studien mehrere Prophylaxen gefunden, nämlich: Herz-Kreislauf, Diabetes, chronische nicht übertragbare Krankheiten, sexuell übertragbare Infektionskrankheiten, parasitäre Krankheiten, Krebs und andere bestehende.

Im Zusammenhang mit Gesetzen und Rechtsgrundlagen für ES zielt es auf eine große Erwartung bei Schülern der frühen Stadien und der High School ab, da der Bildungsprozess nicht im Erwachsenenalter auferlegt wird, sondern im Laufe des Lebens aufgebaut werden kann und zu Wissen wird.

Daher wird als Antwort auf die Frage des richtigen Denkens der Schluss gezogen, dass die ES die Erziehung zur Selbsterkenntnis und Reflexion über ihre eigene Gesundheit sowie das volle Bewusstsein dafür ist, dass etwas schief geht, indem die kritische Reflexion des Subjekts in Bezug auf ihre Gewohnheiten und Präventionsmaßnahmen zugeschrieben wird, wodurch Krankheiten verhindert werden. Es wird durch den Lehrplan der Grundschule sowie durch Regierungsprogramme in Gesundheitszentren angewendet, um die Gesellschaft aufzuklären, um das Bewusstsein für Präventionsmaßnahmen zu schärfen und eine bessere Lebensqualität zu fördern.

\section{VERWEISE}

ABREU JUNIOR, Laerthe de Moraes; CARVALHO, Eliane Vianey de. O discurso médico-higienista no Brasil do início do século XX. Trabalho, Educação e Saúde, v. 10, p. 427-451, 2012.

ALTUVE. R. L. O. La organización de la comunidad como un proceso educativo. Cuadernos de la Escuela de Salud Pública (Caracas) 4:9-28, 1966 
BISCARDE, Daniela Gomes dos Santos; PEREIRA-SANTOS, Marcos; SILVA, Lília Bittencourt. Formação em saúde, extensão universitária e Sistema Único de Saúde (SUS): conexões necessárias entre conhecimento e intervenção centradas na realidade e repercussões no processo formativo. Interface-Comunicação, Saúde, Educação, v. 18, p. 177-186, 2014.

BRASIL. Fundação Nacional de Saúde. Diretrizes de educação em saúde visando à promoção da saúde: documento base - documento I/Fundação Nacional de Saúde -Brasília: Funasa, 2007.

. Ministério da Saúde (MS) Glossário temático: gestão do trabalho e da educação na saúde. Brasília DF, 2012. Disponível em: https://bvsms.saude.gov.br/bvs/publicacoes/glossario_gestao_trabalho_2ed.pdf Acesso em: 03/07/2021.

. Lei; LEI, №. 5.692, DE 11 DE AGOSTO DE 1971. Fixa Diretrizes e Bases para o ensino de, v. 1, p. 6377, 1971.

Lei n. 9.394, de 20 de dezembro de 1996 (1996). Estabelece as diretrizes e bases da educação nacional. Brasília, DF: Ministério da Educação. Disponível em: http://portal.mec.gov.br/seed/arquivos/pdf/tvescola/leis/ lein9394.pdf Acesso em 20 de ago. 2021.

COSTA, Paula Valéria Dias Pena et al. A educação em saúde como ferramenta no combate ao câncer de mama: relato de experiência. Research, Society and Development, v. 9, n. 10, p. e6389108912-e6389108912, 2020.

DE SOUSA GONÇALVES, Romário et al. Educação em saúde como estratégia de prevenção e promoção da saúde de uma unidade básica de saúde. Brazilian Journal of Health Review, v. 3, n. 3, p. 5811-5817, 2020.

DIAS, Ernandes Gonçalves et al. Promoção de saúde na perspectiva da prevenção de doenças parasitárias por meio da educação em saúde com escolares do ensino 
fundamental. Revista de Epidemiologia e Controle de Infecção, v. 8, n. 3, p. 283285, 2018.

ENRIA, G.; STAFFOLANI, C. Contradicciones de los discursos que dificultan la transformación de las prácticas de promoción a la salud. Hacia la promoción de la salud, Calle, v. 15, n. 1, 2010.

FALKENBERG, Mirian Benites et al. Educação em saúde e educação na saúde: conceitos e implicações para a saúde coletiva. Ciência \& Saúde Coletiva, v. 19, p. 847-852, 2014.

FIGUEIREDO, Elisabeth Almeida; ANTUNES, Daniella Cavalcante; DE MIRANDA, Maria Geralda. Políticas públicas de educação em saúde para a prevenção de comorbidades e doenças cardiovasculares. Revista da Seção Judiciária do Rio de Janeiro, [S.I.], v. 23, n. 45, p. 141-160, jul. 2019. ISSN 2177-8337.

GOMES, Ana Cláudia Ferreira et al. Educação em saúde para prevenção do câncer de pênis: relato de experiência. Brazilian Journal of Health Review, v. 2, n. 4, p. 2961-2964, 2019.

JANINI, Janaina Pinto; BESSLER, Danielle; VARGAS, Alessandra Barreto de. Educação em saúde e promoção da saúde: impacto na qualidade de vida do idoso. Saúde em Debate, v. 39, p. 480-490, 2015. LOPES, M. S. V.; SARAIVA, K. R. O.;

LIMA, D. C.; GARCIA, M. P. .; LIMA, E. S. de .; BEZERRA, C. C. . Health education as a tool for the prevention of cardiovascular diseases in the Elderly Health Care Program. Research, Society and Development, [S. I.], v. 9, n. 10, p. e079107382, 2020. DOI: 10.33448/rsd-v9i10.7382. Disponível em: https://rsdjournal.org/index.php/rsd/article/view/7382. Acesso em: 28 aug. 2021.

OBJETIVOS DA ONU - ODS. Objetivo 3. Assegurar uma vida saudável e promover o bem-estar para todas e todos, em todas as idades. Disponível em: <https://gtagenda2030.org.br/ods/ods3/>. Acesso em: 25 ago. 2021 
PIMONT, Rosa Pavone. A educação em saúde: conceitos, definições e objetivos. Boletín de la Oficina Sanitaria Panamericana (OSP); 82 (1), ene. 1977, 1977.

RICCI, Ana Patrícia et al. Infecções sexualmente transmissíveis na gestação: educação em saúde como estratégia de prevenção na atenção básica. Brazilian Journal of Health Review, v. 2, n. 1, p. 565-570, 2019.

SALCI, Maria Aparecida; MEIRELLES, Betina Hörner Schlindwein; SILVA, Denise Maria Guerreiro Vieira da. Educação em saúde para prevenção das complicações crônicas do diabetes mellitus na atenção primáriaa. Escola Anna Nery, v. 22, 2018.

SCHALL, Virgínia T.; STRUCHINER, Miriam. Educação em saúde: novas perspectivas. 1999.

SOUSA, Marta Caires; GUIMARÃES, Ana Paula Miranda; AMANTES, Amanda. A saúde nos documentos curriculares oficiais para o ensino de ciências: da lei de diretrizes e bases da educação à base nacional comum curricular. Revista Brasileira de Pesquisa em Educação em Ciências, p. 129-153, 2019.

SPERANDIO, Naiara; SPERANDIO, Natália Elvira. A educação em saúde enquanto prática transformadora da realidade social. Educação e Saúde: fundamentos e desafios, v. 1, n. 2, p. 7-14, 2018.

XIMENES, L. B. Análise do conceito de promoção da saúde. Texto Contexto Enferm., Florianópolis, v. 19, n. 3, jul./set. 2010, p. 461-468.

\section{ANHANG - REFERENZ FUBNOTE}

7. Menschen, die Teil der Doktrin des Hygienismus waren, einer Doktrin, die in der Mitte des neunzehnten Jahrhunderts geboren wurde, als die Herrscher der Gesundheit und der Moral der Bevölkerung mehr Aufmerksamkeit schenkten. Zu dieser Zeit wurde die Krankheit als ein soziales Phänomen betrachtet, das alle Aspekte des menschlichen Lebens betrifft, durch diesen Kontext entsteht die 
Behandlung von Wasser, Abwasser, Straßenbeleuchtung, um die Pandemien zu kontrollieren oder zu beseitigen (ABREU JUNIOR; EICHE, 2012).

Eingereicht: September 2021.

Genehmigt: November 2021 